\title{
Calor específico a bajas temperaturas de alcoholes sólidos vítreos y cristalinos
}

\author{
C. TALÓN, M. Á. RAMOS, S. VIEIRA \\ Laboratorio de Bajas Temperaturas, Departamento de Física de la Materia Condensada, C-III, Instituto "Nicolás Cabrera", \\ Universidad Autónoma de Madrid, Cantoblanco, Madrid
}

\begin{abstract}
En este trabajo, presentamos y discutimos experimentos de calorimetría a bajas temperaturas que hemos realizado en diferentes vidrios y cristales moleculares (de puentes de hidrógeno). En concreto, hemos medido el calor específico a bajas temperaturas $C_{\mathrm{p}}(T)$ para un conjunto de alcoholes sólidos: etanol normal y completamente deuterado, 1- y 2-propanol, y glicerol. El etanol presenta un muy interesante polimorfismo, con tres fases sólidas diferentes a bajas temperaturas: un cristal estable perfectamente ordenado (monoclínico), un cristal (cúbico) con desorden orientacional o "vidrio orientacional", y el vidrio estructural canónico (amorfo). Midiendo y comparando el calor específico a bajas temperaturas de las tres fases, en el rango de temperaturas del pico bosónico $(2-10 \mathrm{~K})$ así como en el de los estados de tuneleo por debajo de $1 \mathrm{~K}$, hemos podido proporcionar una confirmación cuantitativa de que el "comportamiento vítreo" no es una propiedad exclusiva de los sólidos amorfos. Por otra parte, el propanol es el monoalcohol más sencillo que cuenta con dos estereoisómeros (1- y 2-propanol), lo que nos permite estudiar directamente la influencia de la organización espacial de los átomos en las propiedades universales de los vidrios. Hemos medido el calor específico de ambos isómeros, encontrando una notable diferencia cuantitativa entre ellos. Finalmente, también hemos obtenido el calor específico a bajas temperaturas del glicerol en sus fases vítrea y cristalina.
\end{abstract}

Palabras clave: vidrios, calor especifico, bajas temperaturas, alcoholes, vidrios orientacionales.

Low-temperature specific heat of glassy and crystalline alcohols.

We present and discuss in this work specific-heat experiments at low temperature that we have conducted on different molecular (hydrogenbonded) glasses and crystals. Specifically, we have measured the low-temperature specific heat $C_{\mathrm{p}}(T)$ for a set of solid alcohols: normal and fully-deuterated ethanol, 1- and 2-propanol, and glycerol. Ethanol exhibits a very interesting polymorphism presenting three different solid phases at low temperature: a fully-ordered (monoclinic) crystal, an orientationally-disordered (cubic) crystal or "orientational glass", and the canonical (amorphous) structural glass. By measuring and comparing the low-temperature specific heat of the three phases, in the boson peak range, $2-10 \mathrm{~K}$, as well as in the tunneling-states range, below $1 \mathrm{~K}$, we are able to provide a quantitative confirmation that "glassy behavior" is not an exclusive property of amorphous solids. On the other hand, propanol is the simplest monoalcohol with two different stereoisomers (1and 2-propanol), which allows us to study directly the influence of the spatial rearrangement of atoms on the universal properties of glasses. We have measured the specific heat of both isomers, finding a noteworthy quantitative difference between them. Finally, low-temperature specific-heat data of glassy and crystalline glycerol have also been obtained.

Keywords: glasses, specific heat, low temperatures, alcohols, orientational glasses.

\section{INTRODUCCIÓN}

El comportamiento anómalo de los materiales vítreos a baja temperatura sigue siendo un tema de interés y debate para la comunidad científica. Zeller y Pohl (1) establecieron claramente, hace más de 30 años, que las propiedades térmicas de los sólidos no cristalinos a bajas temperaturas presentan un comportamiento universal, que difiere significativamente del observado en los cristales completamente ordenados. En particular, el calor específico de los vidrios depende linealmente de la temperatura por debajo de $1 \mathrm{~K}$, lo que pronto fue atribuído a la existencia de estados de tuneleo, mientras que por encima de $1 \mathrm{~K} \mathrm{C}_{\mathrm{p}}$ se sigue desviando fuertemente del comportamiento esperado tipo Debye, $T^{3}$, debido a las vibraciones de la red, y muestra un característico máximo ancho en una representación de $C_{\mathrm{p}} / T^{3}$ frente a $T$. (2) Este máximo en $C_{\mathrm{p}} / T^{3}$ está relacionado con el llamado pico bosónico, observado en las espectroscopías vibracionales de dispersión Raman o de neutrones a bajas frecuencias (3), y que consiste en la aparición universal de un máximo ancho en $g(v) / v^{2}$ alrededor de $v \approx 1$ $\mathrm{THz}$, es decir, de un máximo en la densidad de estados vibracionales escalada con la dependencia cuadrática en la frecuencia, esperada según la teoría de Debye.
Por otra parte, el otro gran tema de investigación y debate (3) lo constituye el propio fenómeno de la transición vítrea, que tiene lugar cuando un líquido sobrenfríado se desvía del estado de equilibrio metastable y queda congelado en un sólido desordenado. Aunque, en muchos casos, al sólido no cristalino así formado se le designa indistintamente como vidrio o sólido amorfo, se puede establecer una distinción sutil entre ambos términos, no completamente sinónimos: Un sólido amorfo o no cristalino es aquel material que carece de orden periódico translacional de largo alcance característico de los cristales y que, en algunos casos, debe obtenerse por técnicas alternativas a la del rápido enfriamiento del líquido, ya que no es posible observar el fenómeno de la transición vítrea debido a la fuerte tendencia a la cristalización. Por el contrario, llamaremos vidrio a todo sólido desordenado obtenido sobrenfriando un líquido suficientemente deprisa y que manifiesta pues el fenómeno termodinámico y cinético de la transición vítrea. Como discutiremos a continuación, esta definición de vidrio incluye también a ciertos sistemas que no son sólidos amorfos.

De hecho, un método natural de abordar el problema de la transición vítrea y de la peculiar dinámica de los vidrios a bajas frecuencias 
ha sido estudiar, tanto teórica como experimentalmente, el comportamiento de sólidos cristalinos con algún tipo específico de desorden más o menos controlable y distinto del desorden translacional característico de los sólidos amorfos. Durante muchos años este tipo de estudios se centró en "vidrios orientacionales" obtenidos con cristales mixtos de cianuros alcalinos $u$ otros sistemas similares (4). Estos cristales mixtos poseen una cantidad controlable de desorden orientacional, provocado por las interacciones competitivas entre las posiciones esféricas de los iones halógenos y las elipsoidales de los iones cianuro y que lleva a un estado con desorden orientacional a concentraciones del ion cianuro suficientemente altas.

No obstante, existe otro tipo de "vidrios orientacionales" que presenta un desorden orientacional de tipo dinámico, y que se obtienen mediante un enfriado suficientemente rápido de cristales plásticos (5). Es decir, se evita que el líquido cristalice en la fase de cristal estable (típicamente de baja simetría) congelándose parcialmente en una fase rotora (cristal plástico), donde los centros de masa de las moléculas forman una red cristalina de alta simetría (típicamente cúbica) pero cada molécula puede rotar libremente. Al continuar enfriando este cristal plástico, se pasa por una transición dinámica a la fase de vidrio orientacional, donde el desorden rotacional de las moléculas se convierte en desorden orientacional estático. Este vidrio orientacional es, lógicamente, diferente del verdadero vidrio estructural o amorfo que se obtendría congelando rápidamente el líquido sobrenfriado. Es importante destacar que, a diferencia de los anteriores vidrios orientacionales de cristales mixtos, los obtenidos a partir de cristales plásticos presentan una transición vítrea observable mediante una discontinuidad en el calor específico a una determinada temperatura, y que son materiales de estequiometría homogénea.

En la década de los 70, el grupo de Osaka (6) realizó estudios calorimétricos exhaustivos de un gran número de compuestos de bajo peso molecular, líquidos a temperatura ambiente, encontrando fenómenos de transición vítrea, en el rango $100 \mathrm{~K}$, en todos ellos. En algunos casos, correspondían a las transiciones ordinarias vidrio $\leftrightarrow$ líquido sobrenfriado, mientras que en otras ocasiones se trataba de una transición vidrio orientacional $\leftrightarrow$ cristal plástico. En un caso especialmente interesante, el etanol, podían obtenerse las cuatro fases sólidas del mismo material (cristal estable, cristal plástico, vidrio orientacional y vidrio estructural o amorfo) y observarse las transiciones entre ellas. El etanol se presenta pues como un sistema modelo para estudiar comparativamente las llamadas "propiedades vítreas" en las distintas fases de una misma sustancia.

El siguiente alcohol sencillo monohídrico después del etanol, el propanol, no presenta estas fases intermedias de cristal con desorden orientacional o rotacional, sólo las fases cristalina y amorfa ordinarias. Sin embargo, sí posee la característica de presentar ya dos isómeros, el 1- y el 2-propanol, por lo que parece interesante comparar sus capacidades caloríficas y averiguar si la diferente estructuración de la red de puentes de hidrógeno afecta a aquéllas.

Completaremos nuestro estudio del calor específico a bajas temperaturas de alcoholes sólidos con el glicerol, probablemente el vidrio o líquido molecular sobrenfriado más estudiado en la literatura, en el que también obtendremos y mediremos como referencia fundamental su fase cristalina.

\section{PARTE EXPERIMENTAL}

\subsection{Celda calorimétrica de bajas temperaturas}

El calor específico a bajas temperaturas de las diferentes sustancias se midió empleando una celda experimental $(7,8)$ de tipo cuasiadiabático, especialmente diseñada para muestras que son líquidas a temperatura ambiente y deben solidificarse in situ, dentro del criostato cerrado. La sustancia se introducía en una celda de cobre de paredes delgadas vacía, con una pequeña malla de finos hilos de cobre en su interior para facilitar el rápido equilibrio térmico, que luego se sellaba mecánicamente. La celda llevaba en su exterior un calentador eléctrico y dos termómetros calibrados: uno de germanio para las medidas de bajas temperaturas y un diodo de silicio para medir en el resto del rango de temperaturas.

Se utilizó primero un criostato de ${ }^{4} \mathrm{He}$ para medidas entre 2 y $30 \mathrm{~K}$ $\mathrm{y}$, posteriormente, uno de ${ }^{3} \mathrm{He}$ para poder bajar hasta una temperatura de $0.5 \mathrm{~K}$, encontrando un buen acuerdo entre los datos obtenidos en ambos entornos. Además, el mismo sistema experimental se empleó para caracterizar las diferentes fases y determinar las temperaturas de transición vítreas $T_{\mathrm{g}}$ mediante calorimetría en el rango de 80-200 K. Las diferentes fases estudiadas en cada sustancia (vidrios estructurales o amorfos, vidrios orientacionales y cristales completamente ordenados) fueron obtenidas in situ dentro de la misma celda experimental mediante procesos de enfriamiento rápido, procesos isotérmicos de relajación estructural y cambio de fase, etc.

\subsection{Muestras}

Se empleó etanol completamente deuterado $\mathrm{CD}_{3} \mathrm{CD}_{2} \mathrm{OD}$ (Cambridge Isotope Laboratories, anhidr. $99 \%$ D), sin posterior purificación. Las distintas fases sólidas se prepararon y caracterizaron según el siguiente protocolo (7). Desde la temperatura de fusión del etanol $\left(T_{\mathrm{m}}=159 \mathrm{~K}\right) \mathrm{se}$ enfrió el líquido a velocidades superiores a $-20 \mathrm{~K} / \mathrm{min}$ hasta por debajo de la temperatura de transición vítrea $T_{\mathrm{g}}=95 \mathrm{~K}$. Una vez medida la capacidad calorífica de la fase vítrea a bajas temperaturas, se calentaba el sistema hasta temperaturas de nitrógeno líquido y se medía la capacidad calorífica por el método contínuo $\mathrm{a} \approx+0.2 \mathrm{~K} / \mathrm{min}$, observándose la esperada discontinuidad alrededor de $95 \mathrm{~K}$. Luego, hacia $105-110 \mathrm{~K}$, se producía una transición (exotérmica) de primer orden desde el estado de líquido sobrenfríado al de cristal plástico. Tras enfriarlo y medir el vidrio orientacional a bajas temperaturas, se repetía el proceso anterior de calentamiento, observándose ahora una transición dinámica vidrio orientacional $\leftrightarrow$ cristal plástico también a $95 \mathrm{~K}$ y de magnitud muy similar a la verdadera transición vítrea. En este caso, nada ocurría en el rango 105-110 K, y era al calentar hasta $120 \mathrm{~K}$ aproximadamente cuando se producía la cristalización irreversible a la fase estable (monoclínica). Posteriormente se enfriaba, medía y calentaba esta fase cristalina, que no mostraba lógicamente ninguna discontinuidad termodinámica por debajo de la temperatura de fusión. (7) No se observó ninguna diferencia apreciable para las temperaturas reseñadas entre el etanol normal y el deuterado.

En cuanto al propanol, se utilizaron productos comerciales de 1-propanol $\mathrm{CH}_{3} \mathrm{CH}_{2} \mathrm{CH}_{2} \mathrm{OH}$ (Merck, max. $0.01 \% \mathrm{H}_{2} \mathrm{O}$ ) y de 2-propanol $\mathrm{CH}_{3} \mathrm{CH}(\mathrm{OH}) \mathrm{CH}_{3}$ (Merck, max. $0.05 \% \mathrm{H}_{2} \mathrm{O}$ ). Para obtener las fases vítreas, los líquidos se enfriaron a velocidades del orden de -10 $\mathrm{K} / \mathrm{min}$, desde por encima de sus puntos de fusión $T_{\mathrm{m}}\left(T_{\mathrm{m}}=148 \mathrm{~K}\right.$ para el 1-propanol, $T_{\mathrm{m}}=185 \mathrm{~K}$ para el 2-propanol) hasta sobrepasar las correspondientes transiciones vítreas $T_{\mathrm{g}}\left(T_{\mathrm{g}}=98 \mathrm{~K}\right.$ para el 1-propanol, $T_{\mathrm{g}}=115 \mathrm{~K}$ para el 2-propanol). (9) Las fases cristalinas se obtuvieron calentando las muestras vítreas una vez medidas hasta unos pocos grados por debajo de sus correspondientes temperaturas de fusión.

Para obtener la fase vítrea del glicerol $\mathrm{C}_{3} \mathrm{H}_{5}(\mathrm{OH})_{3}$ (Merck, anhidr., sin purificación posterior) no fue preciso realizar ningún enfriamiento rápido dada su baja tendencia a cristalizar. No obstante, el glicerol posee una fase cristalina ortorrómbica que se pudo preparar (10) mediante un proceso isotérmico de estabilización durante horas entre $T_{\mathrm{g}}$ $=185 \mathrm{~K} \mathrm{y} T_{\mathrm{m}}=291 \mathrm{~K}$. 


\section{RESULTADOS Y DISCUSIÓN}

\subsection{Etanol}

En la Fig. 1, se presentan las medidas de calor específico de las tres fases sólidas a bajas temperaturas del etanol deuterado. Las medidas realizadas para el etanol normal (7) ofrecen resultados muy similares, con diferencias meramente cuantitativas. Como puede observarse, tanto el vidrio convencional (un sólido amorfo) como el orientacional (un cristal desordenado) presentan las mismas características típicas del comportamiento universal de los vidrios a bajas temperaturas: un máximo ancho en $C_{\mathrm{p}} / T^{3}$ alrededor de $6 \mathrm{~K}$ (que se corresponde con el pico bosónico observado por dispersión inelástica de neutrones en esas dos fases "vítreas" del etanol (11)) y un término lineal en el calor específico a muy bajas temperaturas atribuible a los estados de tuneleo (2), que se observa con mayor claridad en la Fig. 2 en una representación $C_{\mathrm{p}} / T$ vs $T$ ${ }^{2}$. Por el contrario, el cristal estable muestra el esperado comportamien- to predicho por la teoría de Debye (el máximo, que también presenta en $C_{p} / T^{3}$, ocurre a temperaturas significativamente mayores y se debe a la dispersión de los fonones acústicos transversales cerca del borde de la zona de Brillouin, como sucede en otros cristales). Para analizar cuantitativamente estos datos de calor específico en los vidrios, se puede emplear el procedimiento postulado en la referencia (12): realizar un ajuste de mínimos cuadrados a un polinomio de grado 2 en la representación $C_{\mathrm{p}} / T$ vs $T^{2}$, en el rango $0<T<(3 / 2) T_{\text {min' }}$ siendo $T_{\min }$ la temperatura donde ocurre el mínimo de $C_{\mathrm{p}} / T^{3}$. Así se obtienen los coeficientes de $C_{\mathrm{p}}=C_{1} T+C_{\text {Debye }} T^{3}+C_{5} T^{5}$, con un sentido físico determinado, y que corresponden a la contribución lineal de los estados de tuneleo, a la cúbica de los "fonones" acústicos de la red, y al término predicho $\propto T^{5}$ por el modelo de potenciales blandos (12), respectivamente. En el caso del cristal estable, sólo existe el término cúbico central, del que se puede extraer directamente su temperatura de Debye. En las Tablas I y II presentamos los resultados de dichos ajustes, junto a otros parámetros básicos, para todos los materiales estudiados en este trabajo.
TABLA I: DATOS CALORIMÉTRICOS PARA LOS VIDRIOS. TEMPERATURAS DE TRANSICIÓN VÍTREA $T_{\mathrm{g}}$ PARA CADA UNO DE LOS VIDRIOS ESTUDIADOS, COEFICIENTES DEL CALOR ESPECÍFICO A BAJAS TEMPERATURAS OBTENIDOS POR AJUSTE DE MÍNIMOS CUADRADOS (VER TEXTO) Y TEMPERATURA DE DebYe, $\theta_{\text {Debye }}$ DEDUCIDA DIRECTAMENTE DEL TÉRMINO CÚBICO. $(\mathrm{EtOH}=$ ETANOL NORMAL. ETOD $=$ ETANOL DEUTERADO).

\begin{tabular}{|l|c|c|c|c|c|}
\cline { 2 - 6 } \multicolumn{1}{c|}{} & $\begin{array}{c}\mathrm{T}_{\mathrm{g}} \\
(\mathrm{K})\end{array}$ & $\mathrm{C}_{1}\left(\mathrm{~mJ} / \mathrm{mol} \cdot \mathrm{K}^{2}\right)$ & $\begin{array}{c}\mathrm{C}_{\text {Debye }} \\
\left(\mathrm{mJ} / \mathrm{mol} \cdot \mathrm{K}^{4}\right)\end{array}$ & $\mathrm{C}_{5}\left(\mathrm{~mJ} / \mathrm{mol} \cdot \mathrm{K}^{6}\right)$ & $\begin{array}{c}\theta_{\text {Debye }} \\
(\mathrm{K})\end{array}$ \\
\hline EtOH -vidrio & 95 & 1.2 & 1.55 & 0.0432 & 224 \\
\hline -v.orientacional & 95 & 1.27 & 1.45 & 0.0288 & 229 \\
\hline EtOD -vidrio & 95 & 1.05 & 1.80 & 0.0572 & 213 \\
\hline -v.orientacional & 95 & 1.13 & 1.72 & 0.0419 & 217 \\
\hline 1-propanol & 98 & 0.424 & 1.77 & 0.0367 & 236 \\
\hline 2-propanol & 115 & 0.516 & 2.54 & 0.0845 & 209 \\
\hline Glicerol & 185 & 0.157 & 0.855 & 0.0139 & 317 \\
\hline
\end{tabular}

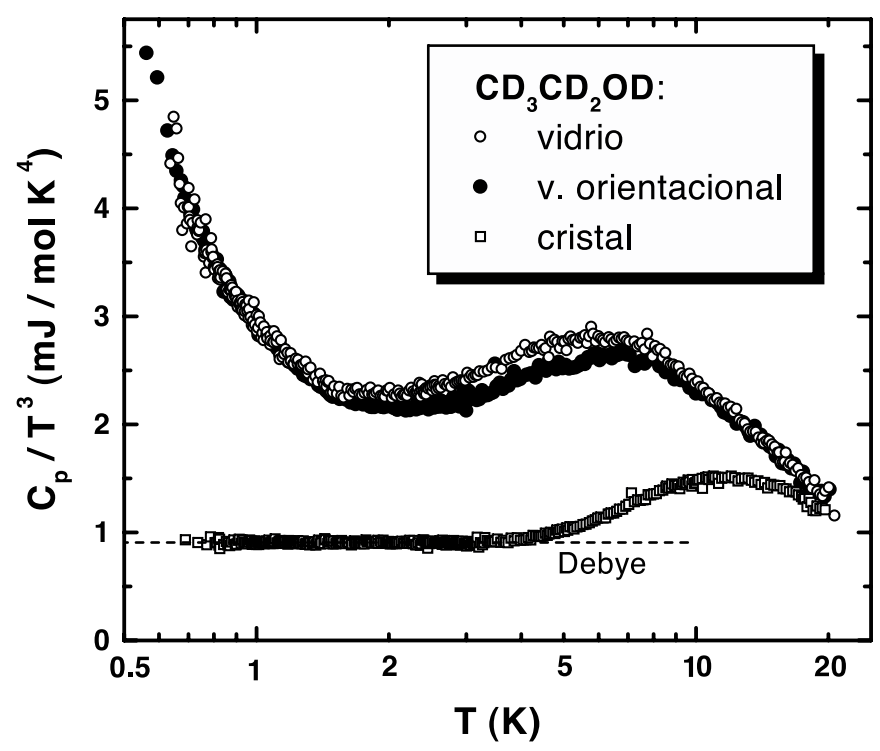

Figura 1: Calor específico $C_{\mathrm{p}} / T^{3}$ frente a la temperatura en escala logarítmica, para las fases de vidrio convencional, vidrio orientacional y cristal estable de etanol completamente deuterado. La línea a trazos indica el límite de la contribución de Debye a las más bajas temperaturas para la fase de cristal estable.
TABla II: DATOS CALORIMÉtricos PARA LOS CRISTALES. TeMPERATURAS DE FUSIÓN $T_{\mathrm{m}}$ DE CADA UNO DE LOS CRISTALES ESTUDIADOS, COEFICIENTES CÚBICOS DE DEBYE DEL CALOR ESPECÍFICO A BAJAS TEMPERATURAS OBTENIDOS MEDIANTE UN AJUSTE LINEAL DE MÍNIMOS CUADRADOS Y CORRESPONDIENTE TEMPERATURA DE DebYe, $\theta_{\text {Debye }}$ DE CADA CRISTAL. $($ EtOH $=$ ETANOL NORMAL. EtOD = ETANOL DEUTERADO).

\begin{tabular}{|c|c|c|c|}
\cline { 2 - 4 } \multicolumn{1}{c|}{} & $\mathrm{T}_{\mathrm{m}}(\mathrm{K})$ & $\mathrm{C}_{\text {Debye }}\left(\mathrm{mJ} / \mathrm{mol} \cdot \mathrm{K}^{4}\right)$ & $\theta_{\text {Debye }}(\mathrm{K})$ \\
\hline EtOH & 159 & 0.766 & 284 \\
\hline EtOD & 159 & 0.906 & 268 \\
\hline 1-propanol & 148 & 1.10 & 277 \\
\hline 2-propanol & 185 & 1.55 & 247 \\
\hline glicerol & 291 & 0.550 & 367 \\
\hline
\end{tabular}

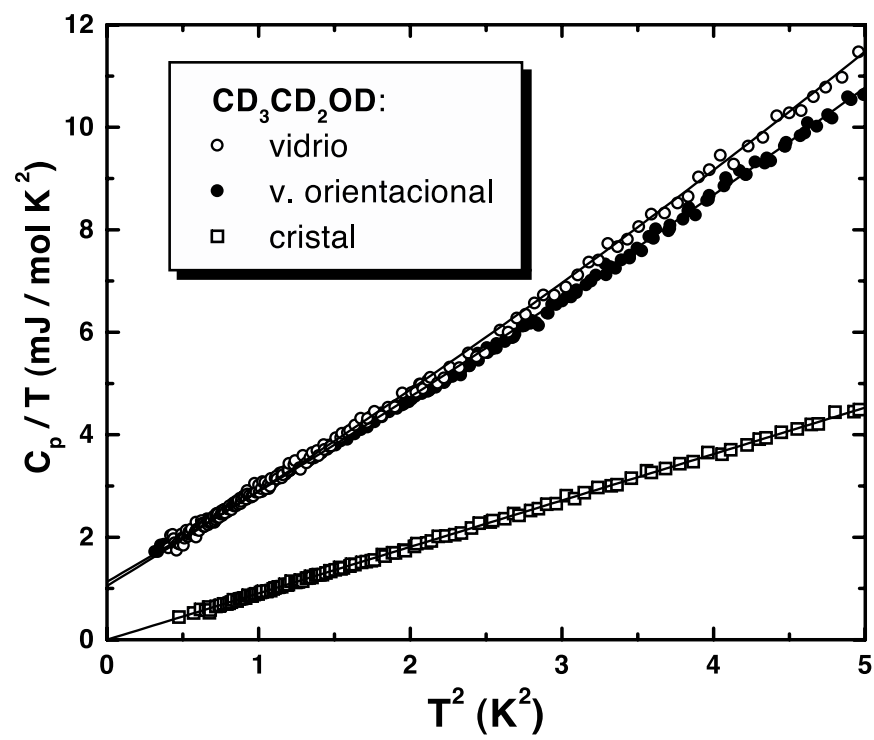

Figura 2: Calor específico $C_{\mathrm{p}} / T^{3}$ frente a $T^{2}$ para las mismas muestras de la Fig. 1. Las líneas contínuas muestran los ajustes de mínimos cuadrados explicados en el texto. El coeficiente lineal de las fases vítreas viene dado por la intersección de las curvas con el eje de ordenadas, siendo nulo para el cristal estable. 
En definitiva, lo más destacable del presente experimento con el etanol, en el que podemos comparar las fases para la misma sustancia, es que dicho comportamiento vítreo del cristal con desorden orientacional no sólo es similar cualitativamente, sino también cuantitativamente, al del verdadero vidrio no cristalino. Este hecho, junto con la idéntica situación relativa a la "transición vítrea", que en ambos casos ocurre a la misma temperatura $\left(T_{\mathrm{g}} \approx 95 \mathrm{~K}\right)$, con una discontinuidad en el calor específico sólo un $\sim 20 \%$ mayor para la transición vidrio $\rightarrow$ líquido sobrenfriado que en la transición vidrio orientacional $\rightarrow$ cristal plástico, nos lleva a concluir que la amorficidad (el desorden topológico translacional) no es el aspecto relevante para entender el estado vítreo, puesto que el desorden orientacional (u otro equivalente en otros sistemas vítreos) ya contiene todos los ingredientes necesarios para dar cuenta -incluso cuantitativamente- del fenómeno de la transición vítrea así como de las "anómalas" propiedades térmicas y vibracionales de los vidrios a bajas temperaturas.

\subsection{Propanol}

En la Fig. 3, se muestran los datos de calor específico a bajas temperaturas para las fases vítrea y cristalina de los dos isómeros del propanol. Estas medidas fueron obtenidas en el criostato de ${ }^{3} \mathrm{He}$ y no se extienden hasta temperaturas tan altas como las alcanzadas previamente empleando el criostato de ${ }^{4} \mathrm{He}$, y que pueden verse en la referencia (9). En cualquier caso, es notable la diferencia en valor absoluto entre los calores específicos de los vidrios correspondientes a ambos isómeros. No obstante, puede observarse también en la Fig. 3 que dicha diferencia relativa existe también entre las respectivas fases cristalinas. Por consiguiente, este gran exceso de calor específico del 2-propanol respecto al 1-propanol puede atribuirse, en su mayor parte, a la apreciable diferencia en las constantes elásticas del sólido que produce que los grupos hidroxilo y, por tanto, los enlaces de puente de hidrógeno que estructuran la red, se hallen en el extremo de la molécula de propanol (1-propanol) o en su centro (2-propanol). De hecho, la estructura cristalina de ambos isómeros de propanol se ha resuelto recientemente (13) mediante difracción de neutrones, encontrándose que la estructura cristalina del 1-propanol es monoclínica (como en el caso del etanol) y la del 2-propanol es triclínica.

\subsection{Glicerol}

Por último, las medidas de calor específico a bajas temperaturas de las fases vítrea y cristalina del glicerol se representan gráficamente en la Fig. 4. De nuevo, el vidrio presenta el comportamiento universal característico de los sólidos no cristalinos, con un máximo en $C_{\mathrm{p}} / T^{3}$ (a una temperatura relativamente alta de $8.7 \mathrm{~K}$ ) y una subida a bajas temperaturas provocada por el término cuasilineal dominante por debajo de $\sim 1 \mathrm{~K}$. En este caso, la contribución elástica tipo Debye de la red al calor específico del vidrio sí es evaluable al disponerse de datos de velocidad del sonido y densidad de masa (10) y se indica también en la Fig. 4. Este valor obtenido a partir de las constantes elásticas medidas en el glicerol vítreo coincide dentro del error experimental con el término cúbico $C_{\text {Debye }}$ obtenido a partir de nuestros ajustes, lo que supone una confirmación del método de análisis postulado (12).

\section{RESUMEN Y CONCLUSIONES}

Hemos realizado medidas de calor específico de diversos alcoholes que son líquidos a temperatura ambiente y tienen la capacidad de formarse en fase vítrea. Se ha prestado una atención especial al etanol puro, tanto hidrogenado como deuterado. El etanol es un material que presenta un rico polimorfismo, mostrando al menos cuatro fases sólidas distintas: un cristal estable (monoclínico), una fase de cristal plástico (bcc) con desorden rotacional -o fase rotora-, un vidrio orientacional obtenido mediante el enfriamiento rápido de dicha fase rotora, y un vidrio estructural o amorfo. El principal resultado ha sido encontrar que el vidrio orientacional (es decir, un cristal con desorden orientacional) posee cualitativa e incluso cuantitativamente el mismo "exceso" de calor específico que el vidrio estructural (es decir, un sólido amorfo), demostrando que la falta de orden traslacional de largo alcance no es un requisito esencial en absoluto para la existencia de las típicas "propiedades vítreas".

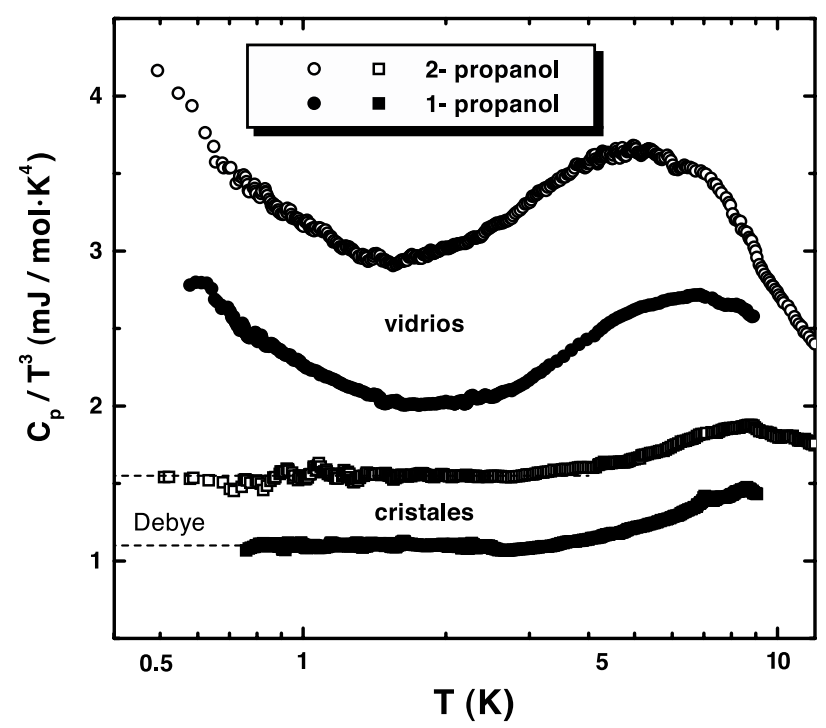

Figura 3: Calor específico $C_{\mathrm{p}} / T^{3}$ frente a la temperatura en escala logarítmica, para las fases de vidrio (círculos) y de cristal (cuadrados) de 1-propanol (símbolos llenos) y 2-propanol (símbolos huecos). Las líneas a trazos indican las contribuciones de Debye para los cristales.

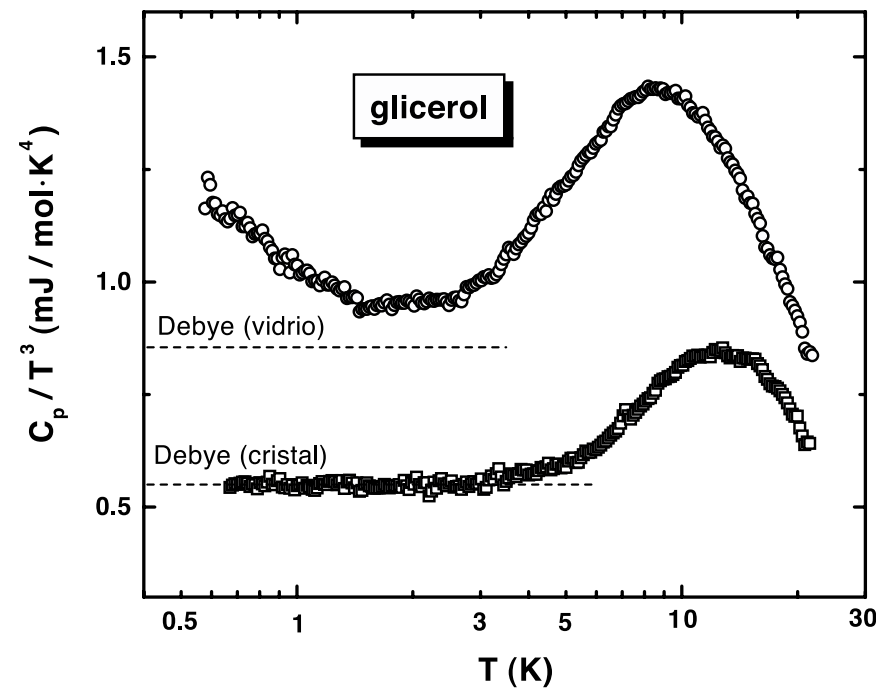

Figura 4: Calor específico $C_{\mathrm{p}} / T^{3}$ frente a la temperatura en escala logarítmica, para las fases de vidrio (círculos) y de cristal (cuadrados) de glicerol. Las líneas a trazos indican las contribuciones respectivas de Debye en el límite de bajas temperaturas, para la fase cristalina y para la vítrea. 
Además del etanol, hemos utilizado la misma técnica para preparar y medir el calor específico del 1-propanol y el 2-propanol, así como del glicerol, estudiándose en todos ellos la fase de cristal estable y la de vidrio convencional. En todos los casos se ha observado, como era de esperar, que la capacidad calorífica de los vidrios es mucho mayor que la de los correspondientes cristales, ya que a su menor temperatura de Debye $\theta_{\text {Debye }}$ (ver Fig. 4) hay que añadir las contribuciones adicionales de los estados de tuneleo y los modos vibracionales de baja frecuencia responsables del llamado pico bosónico. En particular, la gran diferencia de calor específico entre las fases vítreas del 1-propanol y 2-propanol se ha encontrado que es debida principalmente a la gran diferencia de sus contribuciones de Debye respectivas, tal y como también ocurre entre las cristalinas, y no especialmente a los modos vibracionales "en exceso" peculiares de los vidrios.

\section{AGRADECIMIENTOS}

Este trabajo ha sido financiado por el Ministerio de Ciencia y Tecnología dentro del proyecto BFM2000-0035-C02-01.

\section{BIBLIOGRAFÍA}

1. R.C. Zeller, R. O. Pohl. «Thermal Conductivity and Specific Heat of Noncrystalline Solids». Phys. Rev. B 4, 2029-2041 (1971).

2. W. A. Phillips (Ed.). «Amorphous Solids: Low Temperature Properties». Springer, Berlin (Alemania) 1981.
3. Ver, por ejemplo, J. Non-Cryst. Solids 307-310 (2002), Proceedings of the Fourth International Discussion Meeting on Relaxations in Complex Systems, Hersonissos, Creta (Grecia), Junio 2001.

4. U.T. Höchli, K. Knorr, A. Loidl. «Orientational Glasses». Advances in Physics 39, No. 5, 405-615 (1990).

5. J.N. Sherwood (Ed.). «The Plastically Crystalline State (OrientationallyDisordered Crystals)». Wiley, New York (EE.UU.) 1978.

6. H. Suga, S. Seki. «Thermodynamic investigation on glassy states of pure simple compounds». J. Non-Cryst. Solids 16, 171-194 (1974).

7. C. Talón, M.A. Ramos, S. Vieira, G.J. Cuello, F.J. Bermejo, A.Criado, M.L. Senent, S.M. Bennington, H.E. Fischer, H. Schober. «Low-temperature specific heat and glassy dynamics of a polymorphic molecular solid». Phys. Rev. B 58, 745-755 (1998)

8. C. Talón, M.A. Ramos, S. Vieira. «Low-temperature specific heat of amorphous, orientational glass, and crystal phases of ethanol». Phys. Rev. B 66, 012201 (2002).

9. C. Talón, M.A. Ramos, S. Vieira, I. Shmyt'ko, N. Afonikova, A. Criado, G. Madariaga, F.J. Bermejo. «Thermodynamic and structural properties of the two isomers of solid propanol». J. Non-Cryst. Solids 287, 226-230 (2001).

10. C. Talón, Q. W. Zou, M. A. Ramos, R. Villar, S. Vieira. «Low-temperature specific heat and thermal conductivity of glycerol». Phys. Rev. B 65, 012203 (2002).

11. M.A. Ramos, S. Vieira, F.J. Bermejo, J. Dawidowski, H.E. Fisher, H. Schober, M.A. González, C.K. Loong, D. L. Price. «Quantitative Assessment of the Effects of Orientational and Positional Disorder on Glassy Dynamics». Phys. Rev. Lett. 78, 82-85 (1997).

12. M. A. Ramos, C. Talón, S. Vieira. «The Boson peak in structural and orientational glasses of simple alcohols: specific heat at low temperatures». J. Non-Cryst. Solids 307-310, 80-86 (2002).

13. C. Talón, F.J. Bermejo, C. Cabrillo, G.J. Cuello, M.A. González, J.W. Richardson, Jr., A. Criado, M.A. Ramos, S. Vieira, F.L. Cumbrera, L.M. González. «Chemical Isomerism as a Key to Explore Free-Energy Landscapes in Disordered Matter». Phys. Rev. Lett. 88, 115506 (2002).

Recibido: 01.02.03

Aceptado: 30.11 .03 